\title{
Clinical-dosimetric parameters predicting non-classic radiation-induced liver disease in hepatocellular carcinoma patients with Barcelona Clinic Liver Cancer (BCLC) stage A-B: a retrospective study
}

\author{
Li-Wen Huang ${ }^{1}$, Hon-Yi Lin ${ }^{1,2}$, Wen-Yen Chiou ${ }^{1,2}$, Moon-Sing Lee ${ }^{1,2}$, Liang-Cheng Chen ${ }^{1}$, \\ Shih-Kai Hung ${ }^{1,2}$, Nai-Yu Chang ${ }^{3}$ \\ ${ }^{1}$ Department of Radiation Oncology, Dalin Tzu Chi Hospital, Buddhist Tzu Chi Medical Foundation, Chia-Yi, Taiwan; ${ }^{2}$ School of Medicine, Tzu \\ Chi University, Hua-Lien, Taiwan; ${ }^{3}$ ChanDer Clinic, Taipei, Taiwan \\ Contributions: (I) Conception and design: SK Hung, HY Lin; (II) Administrative support: MS Lee, LC Chen; (III) Provision of study materials or \\ patients: SK Hung, HY Lin, WY Chiou; (IV) Collection and assembly of data: LW Huang, NY Chang; (V) Data analysis and interpretation: LW \\ Huang; (VI) Manuscript writing: All authors; (VII) Final approval of manuscript: All authors. \\ Correspondence to: Shih-Kai Hung, MD, PhD. Department of Radiation Oncology, Dalin Tzu Chi Hospital, Buddhist Tzu Chi Medical Foundation, \\ NO. 2, Min-Sheng Road, Dalin Town, Chia-Yi, Taiwan. Email: oncology158@yahoo.com.tw.
}

Background: To identify risk factors relevant to non-classic radiation-induced liver disease (RILD) in hepatocellular carcinoma (HCC) patients with Barcelona Clinic Liver Cancer (BCLC) stage A-B.

Methods: From 2014 to 2018, we retrospectively analyzed 24 HCC patients who were classified with BCLC stage A-B and treated by external beam radiation therapy (EBRT) with volumetric modulated arc therapy (VMAT) technique. We evaluated the correlation between non-classic RILD and clinical-dosimetric parameters. Logistic regression was conducted for multivariate analysis to confirm independent predictors. To assess the predicting ability, the receiver operating characteristic (ROC) curve was used.

Results: Of the 24 irradiated HCC patients with BCLC stage A-B, ten patients had non-classic RILD, with an incidence rate of $41.7 \%$. Identified risk factors for non-classic RILD were Albumin-Bilirubin (ALBI) grade 2-3 and trans-arterial chemoembolization (TACE) gave within 3 months after radiotherapy (RT). The area under the curve (AUC) of the ALBI score in predicting non-classic RILD was 0.786 . The optimal cutoff value of the ALBI score was -2.344, achieving an accuracy rate of 0.793 with sensitivity and specificity of 0.800 and 0.786 , respectively. In multivariate regression analysis, ALBI score more than -2.344 (OR =75.38; 95\% CI: 2.04-2,778.78; P=0.02) was an independent predictor of non-classic RILD.

Conclusions: Applying the ALBI score with a cut-off value of -2.344 is reasonable for clinical use to independently predict the risk of RILD in HCC patients with BCLA stage A-B.

Keywords: Radiotherapy (RT); non-classic radiation-induced liver disease (RILD); hepatocellular carcinoma (HCC)

Received: 10 March 2020; Accepted: 08 April 2020; Published: 30 September 2020.

doi: $10.21037 /$ tro-20-15

View this article at: http://dx.doi.org/10.21037/tro-20-15 


\section{Introduction}

Primary liver cancer is the fifth most common malignancy, and the second most common cause of cancer-related death in Taiwan (1). Primary liver cancer can be mainly categorized into hepatocellular carcinoma (HCC), intrahepatic cholangiocarcinoma, and combination of both. Of these, HCC accounts for $80-90 \%$ of all primary liver cancers (2).

There are many treatment modalities for HCC, and modern radiotherapy (RT) has an emerging role in local control and treating portal vein tumor thrombosis (PVTT). The consensus guideline of the Asia-Pacific Primary Liver Cancer Expert Consortium agreed with the utility and efficacy of external beam radiotherapy (EBRT) in managing patients with early and intermediate stage HCCs, if standard treatment cannot be safely conducted (3).

Radiation-induced liver disease (RILD) is a lifethreatening side effect and limits the utility of EBRT in managing HCC effectively. RILD is separated into "classic" and "non-classic" RILD. Instead of classic RILD, patients with underlying chronic hepatic disease such as viral hepatitis or cirrhosis, tend to develop non-classic RILD (4). The major population of patients with HCC in Taiwan were induced by hepatitis $\mathrm{B}$ virus $(\mathrm{HBV})$ or hepatitis $\mathrm{C}$ virus (HCV) (5), and so do our hospital. Furthermore, by using modern technique like VMAT or IMRT, classic RILD is very rare (6). Many studies demonstrated patients with specific clinical characteristics had higher risks of nonclassic RILD, including patients with Child-Pugh (CP) class $\mathrm{B}$ or $\mathrm{C}(7-10)$, HBV carrier status (11), prior transcatheter arterial chemoembolization (TACE) (8), portal vein tumor thrombosis (PVTT) $(8,10)$, primary tumor stage $(8)$, Albumin-Bilirubin (ALBI) grade (12), and the Cancer of the Liver Italian Program (CLIP) staging system (8). Another study developed a set of dose constraints for liver irradiation (13). However, none of them focused on a patient population of Barcelona Clinic Liver Cancer (BCLC) stage A-B. The present study investigated clinicaldosimetric factors in irradiated HCC patients with BCLC stage A-B for searching potential predicting factors of nonclassic RILD.

We present the following article in accordance with the STROBE reporting checklist (available at http://dx.doi. org/10.21037/tro-20-15).

\section{Methods}

\section{Criteria of patient selection}

From Jun. 2014 to Dec. 2018, patients who were diagnosed with primary HCC and treated with EBRT were retrospectively included in the present study. Exclusive criteria were as follows: age less than 18 years old, EBRT gave after radical surgery, BCLC stage 0 , stage $C$, and stage D. The advanced stage of HCC may have worse preserving liver function, for preventing bias in this study, we excluded patients with BCLC stage C and stage D.

According to the modification of the American Association for the Study of Liver Diseases in 2018 (14), BCLC stages were defined as follows: stage 0 , a single tumor of $\leq 2 \mathrm{~cm}, \mathrm{CP}$ class $\mathrm{A}$, and Eastern Cooperative Oncology Group performance status (ECOG PS) 0-1; stage $\mathrm{A}$, a single tumor $>2 \mathrm{~cm}$ but $\leq 5 \mathrm{~cm}$ or multiple tumors not more than 3 lesions and all $\leq 3 \mathrm{~cm}, \mathrm{CP}$ class $\mathrm{A}-\mathrm{B}$, and ECOG PS $0-1$; stage $\mathrm{B}$, a single tumor $>5 \mathrm{~cm}$ or multiple tumors more than 3 lesions or multiple tumors $>3 \mathrm{~cm}, \mathrm{CP}$ class $\mathrm{A}-\mathrm{B}$, and $\mathrm{ECOG}$ PS $0-1$; stage $\mathrm{C}$, portal invasion, extrahepatic spread, CP class A-B, and ECOG PS 0-2; and stage D, CP class C or ECOG PS 3-4.

\section{Treatments}

All patients received EBRT with the volumetric modulated arc therapy (VMAT) technique for their HCC. Some of them were also treated with TACE, Sorafenib, or both. Respiratory motion management, including deep-expiration breath-hold (DEBH), real-time position management (RPM), 4-dimension CT, and abdominal compression, were applied to the patients according to individual patient's cooperation and condition as well as physician's judgment. The planned target volume (PTV) included gross tumor volume (GTV) with a surrounding margin of 3-5 $\mathrm{mm}$ for covering potential microscopic disease [i.e., clinical target volume (CTV)] and a further margin of 5-10 $\mathrm{mm}$ for covering the uncertainty setup error and tumor motion. The daily dose of 1.8 to 5 Gy per fraction depended on the physician's judgment was prescribed to the PTV up to a median total dose of 57 Gy (range, 15-75 Gy). Furthermore, modified simultaneously integrated boost [i.e., simultaneously integrated inner-escalated boost (SIEB)] (15-19), was performed to irradiate the inner area of GTV, 
with a dose escalation to $110-140 \%$ of prescribed dose. Irradiation was delivered with 6 or $10 \mathrm{MV}$ photons based on tumor size and location. Normal tissue constraints were as follows: mean liver dose <28 Gy, V45 of the bowel bag $<195 \mathrm{cc}$, and mean kidney dose <18 Gy. All patients were irradiated with conventional/short course RT, i.e., one fraction per day and five fractions per week.

\section{Evaluation of hepatic radiation injuries}

During the EBRT course, all patients were monitored by physical examination (PE) and laboratory analysis every week. After completion of EBRT, the tests were done every month, and image studies, e.g., abdominal sonography, CT, or MRI, were performed every 3 months in the first year. After a 1-year follow-up, these examinations were conducted every 3-6 months.

Non-classic RILD $(20,21)$ that commonly encountered for patients with viral hepatitis or cirrhosis demonstrated elevated liver transaminases more than five folds of the upper limit of the normal range, or Common Terminology Criteria for Adverse Events (CTCAE) grade 4 in patients with baseline liver transaminases more than 5 folds of the upper limit of the normal range, or a decline in liver function (i.e., a worse of CP score by 2 or more), in the context of the absence of classic RILD within 3 months after completion of EBRT.

\section{CP classification and ALBI score}

The CP scoring system categorized patients into class A to $\mathrm{C}$ based on five parameters $(22,23)$ : encephalopathy, ascites, serum bilirubin, serum albumin, and prothrombin time. CP class $\mathrm{A}, \mathrm{B}$, and $\mathrm{C}$ were defined as score ranges of 5-6, 7-9, and $10-15$, respectively.

The ALBI score formula was defined as reported previously (24): ALBI score $=\left(\log _{10}\right.$ bilirubin $\left.\times 0.66\right)+$ (albumin $\times-0.085$ ), where bilirubin and albumin are in units of $\mu \mathrm{mol} / \mathrm{L}$ and $\mathrm{g} / \mathrm{L}$, respectively. ALBI grades of 1 , 2 , and 3 were defined as ALBI scores of $\leq-2.60,>-2.60$ to $\leq-1.39$, and $>-1.39$, respectively.

\section{Statistical analysis}

The Chi-square or Student t test was used to compare clinical characteristics and dosimetric parameters between patients with non-classic RILD and those patients without non-classic RILD. Logistic regression was performed to estimate multivariate odds ratios to confirm independent predictors. To avoid statistical uncertainty due to small sample size, Hosmer-Lemeshow test was performed to check the fitness of our logistic regression model ( $\mathrm{P}$ value $=0.383>0.1)$.

Dosimetric parameters and related variables analyzed included normal liver volume, mean liver dose, $\mathrm{V}_{5}, \mathrm{~V}_{10}$, $\mathrm{V}_{15}, \mathrm{~V}_{20}, \mathrm{~V}_{25}, \mathrm{~V}_{30}, \mathrm{~V}_{35}, \mathrm{~V}_{40}, \mathrm{~V}_{45}, \mathrm{~V}_{50}, \mathrm{~V}_{55}$, and $\mathrm{V}_{60}$. Normal liver volume was defined as total liver volume minus GTV volume, and mean liver dose represented mean normal liver dose. Note that $V_{5}$ is defined as the percentage of the total normal liver volume that is irradiated with a dose of more than or equal to 5 Gy (25). Similarly, V10-V60 were defined in the same way.

Survival was estimated from the date of initiation of treatment to the date of the last follow-up visit by the Kaplan-Meier method. The receiver operating characteristic (ROC) curve was further illustrated for evaluating the ALBI score in predicting the risk of non-classic RILD, as previously reported (12). The cut-off point was determined to be the value at the maximum value of the Youden Index (26). SPSS software was used for conducting statistical analysis, with a significant level set at $\mathrm{P}<0.05$.

\section{Statement of etbics approval}

The study was conducted in accordance with the Declaration of Helsinki (as revised in 2013). The study was approved by institutional review board of Dalin Tzu Chi Hospital (NO.: B10704005) and informed consents were waived by the institutional review board due to no additional therapies were need in this retrospective study.

\section{Results}

From Jun. 2014 to Dec. 2018, a total of 169 patients diagnosed with HCC with status post EBRT were included. A total of 145 patients were excluded due to following etiologies: BCLC stage C-D $(n=118)$ and EBRT gave after surgery $(n=27)$. As a result, 24 patients were included for analysis. Their clinical characteristics and dosimetric data were collected. All patients were technically unresectable or medically inoperable and were unsuitable for radiofrequency ablation (RFA) or percutaneous ethanol injection (PEI).

According to the BCLC staging system $(22,23)$, there were 5 patients with BCLC stage A and 19 patients with BCLC stage $\mathrm{B}$, and no portal vein thrombosis was detected. The mean age was 66 years (range, $48-86$ years), and the male-to- 
female ratio was 1.18. All patients had a good performance status (i.e., ECOG PS 0 or 1). Status of virus hepatitis for the included patients was as follows: HBV carrier $(n=7), \mathrm{HCV}$ infection ( $\mathrm{n}=17)$, both HBV and HCV $(\mathrm{n}=1)$, and none $(\mathrm{n}=1)$. When classified by CP classification for liver cirrhosis, 17 patients (71\%) were CP class A, and 7 patients (29\%) were $\mathrm{CP}$ class B. For liver function preserve at the time of EBRT, distribution of ALBI grade was as follows: ALBI grade 1 $(\mathrm{n}=11)$, grades $2(\mathrm{n}=11)$, and grade $3(\mathrm{n}=2)$.

All patients received EBRT with the VMAT technique. Respiratory motion management was applied accordingly, in terms of DEBH $(n=2), \operatorname{RPM}(n=3), 4 D C T(n=7)$, and abdominal compression $(\mathrm{n}=2)$. The mean PTV volume was $231.1 \mathrm{~mL}$ (range, $32.7-750 \mathrm{~mL}$ ). The mean equivalent dose in 2-Gy fraction $\left(\mathrm{EQD}_{2}\right)$ at $\alpha / \beta$ of 10 to PTV was $59.9 \mathrm{~Gy}_{10}$. Four patients had TACE after RT within 3 months, and 8 patients had TACE before RT within 3 months. Only one patient received Sorafenib before $\mathrm{RT}$, and the duration between the day of the last dose of Sorafenib and the day of RT initiation was 33 days. Three patients received Sorafenib during and after RT, and no patient received it only after completion of RT.

\section{Follow-up and outcome}

The median follow-up time was 15.0 months (range, 1.2-51.7 months) after completion of RT. Overall survival rates at $1,2,3$, and 5 years were $74.1 \%, 38.9 \%, 38.9 \%$, and $25.9 \%$, respectively, with a median survival of 16.1 months. Ten patients had non-classic RILD with an incidence rate of $41.7 \%$.

By using the Chi-square test, we observed two factors, i.e., ALBI grade and the timing of TACE after RT, were significantly correlated with the occurrence of non-classic RILD (Table 1). The incidences of non-classic RILD were $61.54 \%$ and $18.18 \%$ for patients with ALBI grade $2-3$ and grade $1(\mathrm{P}=0.047)$, respectively. The incidences of nonclassic RILD were $100 \%$ and $30 \%$ for patients received TACE within 3 months after RT and for those patients received TACE more than 3 months after RT or no TACE $(\mathrm{P}=0.020)$, respectively.

ALBI score was statistically significantly different between the two groups of patients with and without nonclassic RILD (i.e., $-1.99 \pm 0.79$ vs. $-2.62 \pm 0.47, \mathrm{P}=0.023$; Table 2). However, $\mathrm{PTV} \mathrm{EQD}_{2}$ (at $\alpha / \beta$ of 10 ), PTV volume, boost volume, normal liver volume, mean liver dose, $\mathrm{V}_{5}$, $\mathrm{V}_{10}, \mathrm{~V}_{15}, \mathrm{~V}_{20}, \mathrm{~V}_{25}, \mathrm{~V}_{30}, \mathrm{~V}_{35}, \mathrm{~V}_{40}, \mathrm{~V}_{45}, \mathrm{~V}_{50}, \mathrm{~V}_{55}$, and $\mathrm{V}_{60}$ showed no significant difference between the two groups. The predicting ability of the ALBI score for the risk of nonclassic RILD was further evaluated by using the ROC curve, with an AUC value of $0.786(\mathrm{P}=0.019$; Figure 1).

The variate, TACE after RT within 3 months, had ever tested univariate logistic regression analysis. However, this variate did not show significant impact on the non-classic RILD. Hence, the TACE after RT within 3 months did not include in the multivariate analysis. In our multivariate analysis, we used stepwise selection and followed the historical studies to choose variates for the multivariate analysis. The HBV carrier (11) and CP classification (7-10) had been reported as risk factors of non-classic RILD. The HCV carrier had not been reported as a risk factor of non-classic RILD, but it is harmful to the liver function and has high prevalence in Taiwan (5). Therefore, they were selected in the multivariate analysis. The gender and age were included in the multivariate analysis as baseline characteristics. ROC curve analysis identified a cutoff point of -2.344 for ALBI score achieved an accuracy rate of 0.793 for predicting the risk of non-classic RILD, with a sensitivity of 0.8 and a specificity of 0.786 . Remarkably, logistic regression showed that ALBI score more than -2.344 (OR, 14.67; 95\% CI, 1.97-109.20; P=0.01) demonstrates a bigger odds ratio than that of ALBI score itself (OR, 5.57; 95\% CI, 1.03-30.10; $\mathrm{P}=0.046)$ and ALBI grade $2-3(\mathrm{OR}, 7.20 ; 95 \% \mathrm{CI}, 1.08-47.96 ; \mathrm{P}=0.04)$ for predicting non-classic RILD. Furthermore, multivariate regression confirmed ALBI score more than -2.344 as an independent predictor for non-classic RILD (OR, 75.38; 95\% CI, 2.04-2,778.78; $\mathrm{P}=0.02$; Table 3).

\section{Discussion}

BCLC stage is easily applied to stratify HCC patients for clinical management. According to a recent review paper (27), the predicted survival of patients with BCLC stage A-B was 2.5-5 years. However, in the present study, the observed patient survival was shorter. The reason for this discrepancy was that the survival time of the present study was calculated from the date of initiation of RT, not the day at diagnosis that applied in other studies. Based on the consensus guideline of the Asia-Pacific Primary Liver Cancer Expert consortium (3), RT is indicated for the patients diagnosed with BCLC stage A-B and not suitable for standard treatment, e.g., surgical resection and RFA. In the present study, all patients had received at least one therapy before RT, and they were referred to receive RT for consolidation, disease progress, or recurrence. 
Table $1 \chi^{2}$ analysis of clinical characteristics in correlation with non-classic RILD for 24 HCC patients who were classified with BCLC stage A-B and treated with EBRT

\begin{tabular}{|c|c|c|c|c|c|}
\hline Variable & No. of case & No. with non-classic RILD & No. without non-classic RILD & $\chi^{2}$ & $P$ value \\
\hline Male & 13 & $4(30.77 \%)$ & $9(69.23 \%)$ & & \\
\hline Female & 11 & $6(54.55 \%)$ & $5(45.45 \%)$ & & \\
\hline BCLC stage & & & & 1.22 & 0.358 \\
\hline Stage B & 19 & $9(47.37 \%)$ & $10(52.63 \%)$ & & \\
\hline HBV or HCV infection & & & & 0.745 & 1.000 \\
\hline Negative & 1 & $0(0.00 \%)$ & $1(100.00 \%)$ & & \\
\hline Positive & 23 & $10(43.48 \%)$ & $13(56.52 \%)$ & & \\
\hline Positive & 7 & $2(28.57 \%)$ & $5(71.43 \%)$ & & \\
\hline HCV infection & & & & 0.697 & 0.653 \\
\hline Negative & 7 & $2(28.57 \%)$ & $5(71.43 \%)$ & & \\
\hline Positive & 17 & $8(47.06 \%)$ & $9(52.94 \%)$ & & \\
\hline CP classification & & & & 1.386 & 0.408 \\
\hline Class A & 13 & $4(30.77 \%)$ & $9(69.23 \%)$ & & \\
\hline Class B & 11 & $6(54.55 \%)$ & $5(45.45 \%)$ & & \\
\hline ALBI & & & & 4.608 & 0.047 \\
\hline TACE after RT & & & & 6.72 & 0.020 \\
\hline None or $>3$ months & 20 & $6(30.00 \%)$ & $14(70.00 \%)$ & & \\
\hline Within 3 months & 4 & $4(100.00 \%)$ & $0(0.00 \%)$ & & \\
\hline Sorafenib during RT & & & & 2.449 & 0.239 \\
\hline No & 21 & $10(47.62 \%)$ & $11(52.38 \%)$ & & \\
\hline Yes & 3 & $0(0.00 \%)$ & $3(100.00 \%)$ & & \\
\hline Chinese herbal medicine & & & & 0.229 & 0.665 \\
\hline$<1$ month & 18 & 7 (38.89\%) & $11(61.11 \%)$ & & \\
\hline$\geq 1$ month & 6 & $3(50.00 \%)$ & $3(50.00 \%)$ & & \\
\hline
\end{tabular}

ALBI, Albumin-Bilirubin; BCLC, Barcelona Clinic Liver Cancer; CP classification, Child-Pugh classification; EBRT, external beam radiation therapy; HBV, hepatitis B virus; HCV, hepatitis C virus; RILD, radiation-induced liver disease; RT, radiotherapy; TACE, transarterial chemoembolization. 
Table 2 Mean values of dosimetric parameters and ALBI score in patients with and without non-classic RILD

\begin{tabular}{|c|c|c|c|}
\hline Variable & With non-classic RILD & Without non-classic RILD & $\mathrm{P}$ \\
\hline Mean liver dose (Gy) & $12.2 \pm 6.8$ & $13.4 \pm 5.3$ & 0.619 \\
\hline$V_{5}(\%)$ & $69.6 \pm 26.9$ & $60.4 \pm 22.4$ & 0.371 \\
\hline $\mathrm{V}_{10}(\%)$ & $49.2 \pm 25.7$ & $47.0 \pm 18.8$ & 0.807 \\
\hline $\mathrm{V}_{20}(\%)$ & $25.6 \pm 15.0$ & $29.3 \pm 15.3$ & 0.556 \\
\hline $\mathrm{V}_{25}(\%)$ & $16.3 \pm 9.2$ & $21.9 \pm 12.5$ & 0.244 \\
\hline $\mathrm{V}_{30}(\%)$ & $11.4 \pm 7.3$ & $15.9 \pm 10.0$ & 0.247 \\
\hline $\mathrm{V}_{35}(\%)$ & $7.1 \pm 5.7$ & $11.2 \pm 7.6$ & 0.168 \\
\hline $\mathrm{V}_{50}(\%)$ & $1.3 \pm 2.5$ & $2.6 \pm 2.6$ & 0.238 \\
\hline $\mathrm{V}_{55}(\%)$ & $0.7 \pm 1.5$ & $1.3 \pm 1.4$ & 0.293 \\
\hline $\mathrm{V}_{60}(\%)$ & $0.3 \pm 0.8$ & $0.7 \pm 1.3$ & 0.355 \\
\hline PTV EQD $2\left(G y_{10}\right)$ & $51.3 \pm 22.3$ & $66.0 \pm 15.3$ & 0.069 \\
\hline ALBI score & $-1.99 \pm 0.79$ & $-2.62 \pm 0.47$ & 0.023 \\
\hline
\end{tabular}

Data are shown as mean \pm standard deviation. ALBI, Albumin-Bilirubin; RILD, radiation-induced liver disease; $\mathrm{PTV}_{\mathrm{EQD}}$, planned target volume equivalent dose in 2-Gy fractionation at $\alpha / \beta$ of $10 .{ }^{*}$, note that 'Vdose' is the percentage of total normal liver volume receiving irradiation doses of more than or equal to the specified dose level. For example, ' $V_{5}$ ' is the percentage of total normal liver volume receiving an irradiation dose of $\geq 5$ Gy.

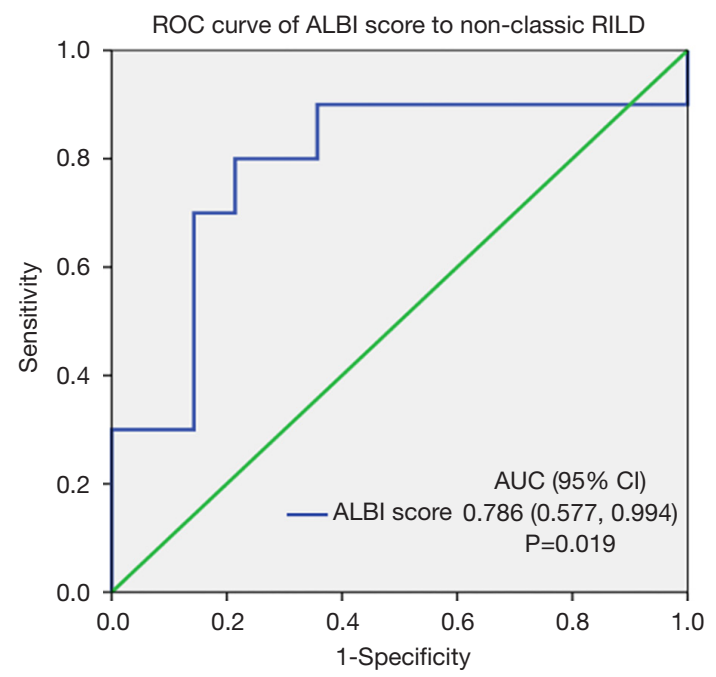

Figure 1 ROC curve and corresponding AUC for ALBI in predicting the risk of non-classic RILD. ALBI, Albumin-Bilirubin; AUC, area under the curve; RILD, radiation-induced liver disease; ROC, receiver operating characteristic.

\section{The incidence of RILD varied from}

In the past, RT demonstrated limited effectiveness in managing HCC patients, mainly due to the constraint of normal liver tolerance. This constraint limits the indication of RT for HCC patients in palliation. With the advances of EBRT techniques, including IMRT, VMAT, and stereotactic ablative radiotherapy/stereotactic body radiation therapy (SABR/SBRT), high doses of RT can be delivered accurately to gain high local control, with a cost of limited toxicities. Several phase III studies have proven the efficacy of these modern precise RT (28-30). As a result, the indication of RT is widely expanded for managing patients with HCC.

Historically, the incidence rate of RILD among patients who received hepatic radiation was ranged from $6 \%$ to $66 \%$ $(20,31,32)$. The wide range of RILD incidence rate was related to the definition of RILD, individual liver function reserve, and RT technique. By using modern irradiation 
Table 3 Univariate and multivariate analysis of potential predictors of non-classic RILD

\begin{tabular}{|c|c|c|c|c|}
\hline Variable & \multicolumn{2}{|c|}{ Univariate analysis } & \multicolumn{2}{|c|}{ Multivariate analysis } \\
\hline Gender & $2.70(0.51-14.37)$ & 0.244 & $4.75(0.18-123.30)$ & 0.35 \\
\hline Age & $1.00(0.93-1.08)$ & 0.956 & $1.05(0.89-1.24)$ & 0.57 \\
\hline HBV carrier & $0.45(0.07-3.00)$ & 0.409 & $0.47(0.01-36.64)$ & 0.73 \\
\hline CP class $\mathrm{B}$ & $2.44(0.40-14.75)$ & 0.33 & $0.30(0.02-5.11)$ & 0.40 \\
\hline ALBI score more than -2.344 & $14.67(1.97-109.2)$ & 0.009 & $75.38(2.04-2,778.78)$ & 0.02 \\
\hline
\end{tabular}

ALBI, Albumin-Bilirubin; CP class B, Child-Pugh class B; HBV, hepatitis B virus; HCV, hepatitis C virus; RILD, radiation-induced liver disease.

technique, focal RT to the tumor has replaced the outdated technique of whole liver irradiation, and a new type of RILD has been discovered $(20,21)$. The non-classic RILD is the primary pattern of RILD in patients with hepatitis or cirrhosis and treated with modern techniques of RT. A Korean study (33) reported that the incidence rates of classic and non-classic RILD were $12 \%$ and $35.9 \%$, respectively. Among the group of non-classic RILD, 66.7\% of patients developed a worse of CP score by $\geq 2$ points. In the present study, we reported a slightly higher rate of $41.7 \%$ in developing non-classic RILD, with $70 \%$ of patients experienced worsening CP scores by $\geq 2$ points.

Due to high mortality rates of non-classic RILD (33), many studies tried to figure out risk factors, including dosimetric parameters, which influence liver toxicities (7-13). However, the risk of non-classic RILD is still poorly predictable in patients with underlying liver disease (4).

ALBI grade was developed in 2015 (24) and was reported to be a better prognostic factor than that of CP score in HCC cancer patients (12). In the present study, non-classic RILD was statistically significantly associated with ALBI grade $(\mathrm{P}=0.047)$, but not the $\mathrm{CP}$ score $(\mathrm{P}=0.408)$. Patients with ALBI grade 1 had a lower non-classic RILD rate than that with ALBI grade $2-3$ (18.18\% vs. $61.54 \%, \mathrm{P}=0.047)$. The AUC of ALBI score in predicting non-classic RILD was 0.786 . The optimal cut-off value of the ALBI score was -2.344 with accuracy, sensitivity, and specificity of $0.793,0.8$, and 0.786 , respectively. Remarkably, multivariate regression confirmed ALBI score more than $-2.344(\mathrm{OR}=75.38$ with 95\% CI: 2.04-2,778.78; $\mathrm{P}=0.02$ ) as an independent predictor for non-classic RILD (Table 3).

A phase III study conducted by Yoon et al. (28) demonstrated that TACE combined with RT was safe and beneficial to survival in HCC patients with PVTT.
However, when compared with Yoon's study (28), the present study showed that TACE conducted within 3 months after RT was a risk factor of non-classic RILD $(\mathrm{P}=0.020)$. The reason causing the difference may be that all patients in the Yoon's study were CP class A with a pretreatment albumin of $\geq 3.3 \mathrm{~g} / \mathrm{dL}$. This good pre-treatment liver function can prevent non-classic RILD in patients who received a combined modality of TACE plus RT.

In a study reported from China (13), $9 \%$ and $56 \%$ of patients with CP class A and B developed RILD, respectively. Among patients with CP class A, normal liver volume and several dosimetric parameters demonstrated abilities to predict the risk of RILD. As a result, a set of liver dose constraints was established. However, their population included only patients with cT3-4 tumors that were defined based on the American Joint Committee on Cancer TNMstaging system (the $5^{\text {th }}$ edition, 1997). In contrast, the present study investigated in patients with BCLC stage A-B, and our results showed that neither normal liver volume nor dosimetric parameters were statistically significantly different between patients with and without non-classic RILD.

This study analyzed several clinical characteristics and dosimetric parameters. Surprisingly, we found the risk factors that predicted non-classic RILD had lots of differences to several previous studies (7-13). The reasons causing these differences may be due to different patient populations. In the present study, we investigated HCC patients with BCLC stage A-B comparing to major patient's components of BCLC stage $\mathrm{C}-\mathrm{D}$ in all the other studies, and only 24 patients were analyzed.

To the best of the authors' knowledge, this is the first study analyzing the risk factors of non-classic RILD in HCC patients with BCLC stage A-B. ALBI grades of 2-3 and TACE within 3 months after RT had significantly 
higher incidences of non-classic RILD. The ALBI score was an acceptable predictor for non-classic RILD with a cut-off point value of -2.344 . These findings can be applied to clinical practice in managing HCC patients with BCLC stage A-B. A patient with HCC BCLC stage A or $B$ having ALBI score more than -2.344 should not receive conventional/short course RT, except under suggestion of multidiscipline cancer meetings, or choose better or more advanced RT techniques, like SBRT/respiratory motion management, etc.

Because of small sample size, further large multicenter study is needed. Besides, because majority of our patient had HBV or HCV infection, the generalization of our results among different populations and institutions might be questionable. Further study in HBV/HCV non-epidemic area, like Europe or U.S., should be performed to decide their cut-off value of ALBI score for predicting non-classic RILD in their population.

Due to the limited patient number, comparing to other studies, most results of the parameters in Table 1 and Table 2 showed no significant difference, even the CP classification. We predicted that $\mathrm{CP}$ classification and dosimetric data might be significant if the case number expanded. As a result, further prospective studies with a massive case number are needed to validate the hypothesis.

\section{Conclusions}

In HCC patients with BCLC stage A-B, the ALBI score is an acceptably independent factor for predicting non-classic RILD with a cut-off value of -2.344 . However, mainly due to the limitation of the small case number, a further sizeable prospective study should be warranted to confirm our results.

\section{Acknowledgments}

We would also like to show our gratitude to the HsuanJu Yang and Ming-Hsien Yeh, Buddhist Dalin Tzu Chi Hospital for sharing their pearls of wisdom with us during the course of this research.

Funding: This study is supported by Dalin Tzu Chi Hospital (No: DTCRD108-I-15).

\section{Footnote}

Provenance and Peer Review: This article was commissioned by the Guest Editors (Chen-Hsi Hsieh, Hsin-Hua Nien, and Yu-Ming Huang) for the series "Precision Medicine for Cancer" published in Therapeutic Radiology and Oncology. The article has undergone external peer review.

Reporting Checklist: The authors have completed the STROBE reporting checklist. Available at http://dx.doi. org/10.21037/tro-20-15

Conflicts of Interest: All authors have completed the ICMJE uniform disclosure form (available at http://dx.doi. org/10.21037/tro-20-15). The series "Precision Medicine for Cancer" was commissioned by the editorial office without any funding or sponsorship. Shih-Kai Hung serves as an unpaid editorial board member of Therapeutic Radiology and Oncology from Apr 2020 to Mar 2022. The authors have no other conflicts of interest to declare.

Ethical Statement: The authors are accountable for all aspects of the work in ensuring that questions related to the accuracy or integrity of any part of the work are appropriately investigated and resolved. The study was conducted in accordance with the Declaration of Helsinki (as revised in 2013). The study was approved by institutional review board of Dalin Tzu Chi Hospital (NO.: B10704005) and informed consents were waived by the institutional review board due to no additional therapies were need in this retrospective study.

Open Access Statement: This is an Open Access article distributed in accordance with the Creative Commons Attribution-NonCommercial-NoDerivs 4.0 International License (CC BY-NC-ND 4.0), which permits the noncommercial replication and distribution of the article with the strict proviso that no changes or edits are made and the original work is properly cited (including links to both the formal publication through the relevant DOI and the license). See: https://creativecommons.org/licenses/by-nc-nd/4.0/.

\section{References}

1. 105 nian yanzheng dengji baogao. Available online: https://www.hpa.gov.tw/Pages/Detail. aspx? nodeid $=269 \&$ pid $=10227$

2. Yang JD, Hainaut P, Gores GJ, et al. A global view of hepatocellular carcinoma: trends, risk, prevention and management. Nat Rev Gastroenterol Hepatol 2019;16:589-604.

3. Park HC, Yu JI, Cheng JC, et al. Consensus for 
Radiotherapy in Hepatocellular Carcinoma from The 5th Asia-Pacific Primary Liver Cancer Expert Meeting (APPLE 2014): Current Practice and Future Clinical Trials. Liver Cancer 2016;5:162-74.

4. Munoz-Schuffenegger P, Ng S, Dawson LA. RadiationInduced Liver Toxicity. Semin Radiat Oncol 2017;27:350-7.

5. Chen DS. Hepatocellular carcinoma in Taiwan. Hepatol Res 2007;37 Suppl 2:S101-5.

6. Koay EJ, Owen D, and Das P. Radiation-Induced Liver Disease and Modern Radiotherapy. Semin Radiat Oncol 2018;28:321-31.

7. Xu ZY, Liang SX, Zhu J, et al. Prediction of radiationinduced liver disease by Lyman normal-tissue complication probability model in three-dimensional conformal radiation therapy for primary liver carcinoma. Int J Radiat Oncol Biol Phys 2006;65:189-95.

8. Liang SX, Zhu XD, Xu ZY, et al. Radiation-induced liver disease in three-dimensional conformal radiation therapy for primary liver carcinoma: the risk factors and hepatic radiation tolerance. Int J Radiat Oncol Biol Phys 2006;65:426-34.

9. Lee IJ, Seong J, Shim SJ, et al. Radiotherapeutic parameters predictive of liver complications induced by liver tumor radiotherapy. Int J Radiat Oncol Biol Phys 2009;73:154-8.

10. Huang Y, Chen SW, Fan CC, et al. Clinical parameters for predicting radiation-induced liver disease after intrahepatic reirradiation for hepatocellular carcinoma. Radiat Oncol 2016;11:89.

11. Cheng JC, Wu JK, Lee PC, et al. Biologic susceptibility of hepatocellular carcinoma patients treated with radiotherapy to radiation-induced liver disease. Int J Radiat Oncol Biol Phys 2004;60:1502-9.

12. Ho CHM, Chiang CL, Lee FAS, et al. Comparison of platelet-albumin-bilirubin (PALBI), albumin-bilirubin (ALBI), and child-pugh (CP) score for predicting of survival in advanced hcc patients receiving radiotherapy (RT). Oncotarget 2018;9:28818-29.

13. Liang SX, Zhu XD, Xu ZY, et al. Radiation-induced liver disease in three-dimensional conformal radiation therapy for primary liver carcinoma: the risk factors and hepatic radiation tolerance. Int J Radiat Oncol Biol Phys 2006;65:426-34.

14. Marrero JA, Kulik LM, Sirlin CB, et al. Diagnosis, Staging, and Management of Hepatocellular Carcinoma: 2018 Practice Guidance by the American Association for the Study of Liver Diseases. Hepatology 2018;68:723-750.

15. Lin YH, Hung SK, Chiou WY, et al. Significant symptoms alleviation and tumor volume reduction after combined simultaneously integrated inner-escalated boost and volumetric-modulated arc radiotherapy in a patient with unresectable bulky hepatocellular carcinoma: A care-compliant case report. Medicine (Baltimore) 2016;95:e4717.

16. Nomiya T, Akamatsu H, Harada M, et al. Modified simultaneous integrated boost radiotherapy for an unresectable huge refractory pelvic tumor diagnosed as a rectal adenocarcinoma. World J Gastroenterol 2014;20:18480-6.

17. Nomiya T, Akamatsu H, Harada M, et al. Modified simultaneous integrated boost radiotherapy for large retroperitoneal malignant tumor: A case report. Oncol Lett 2015;9:2520-4.

18. Nomiya T, Akamatsu H, Harada M, et al. Modified simultaneous integrated boost radiotherapy for unresectable locally advanced breast cancer: preliminary results of a prospective clinical trial. Clin Breast Cancer 2015;15:161-7.

19. Crane CH, Koay EJ. Solutions that enable ablative radiotherapy for large liver tumors: Fractionated dose painting, simultaneous integrated protection, motion management, and computed tomography image guidance. Cancer 2016;122:1974-86.

20. Pan CC, Kavanagh BD, Dawson LA, et al. Radiationassociated liver injury. Int J Radiat Oncol Biol Phys 2010;76:S94-100.

21. Cheng JC, Wu JK, Huang CM, et al. Radiation-induced liver disease after radiotherapy for hepatocellular carcinoma: clinical manifestation and dosimetric description. Radiother Oncol 2002;63:41-5.

22. Child CG, Turcotte JG. Surgery and portal hypertension. Major Probl Clin Surg 1964;1:1-85.

23. Pugh RN, Murray-Lyon IM, Dawson JL, et al. Transection of the oesophagus for bleeding oesophageal varices. Br J Surg 1973;60:646-9.

24. Johnson PJ, Berhane S, Kagebayashi C, et al. Assessment of liver function in patients with hepatocellular carcinoma: a new evidence-based approach-the ALBI grade. J Clin Oncol 2015;33:550-8.

25. Wang $W, \mathrm{Xu} Y$, Schipper $M$, et al. Effect of normal lung definition on lung dosimetry and lung toxicity prediction in radiation therapy treatment planning. Int J Radiat Oncol Biol Phys 2013;86:956-63.

26. Youden WJ. Index for rating diagnostic tests. Cancer 1950;3:32-5.

27. Forner A, Reig M, Bruix J. Hepatocellular carcinoma. 
Lancet 2018;391:1301-14.

28. Yoon SM, Ryoo BY, Lee SJ, et al. Efficacy and Safety of Transarterial Chemoembolization Plus External Beam Radiotherapy vs Sorafenib in Hepatocellular Carcinoma With Macroscopic Vascular Invasion: A Randomized Clinical Trial. JAMA Oncol 2018;4:661-9.

29. Wei X, Jiang Y, Zhang X, et al. Neoadjuvant ThreeDimensional Conformal Radiotherapy for Resectable Hepatocellular Carcinoma With Portal Vein Tumor Thrombus: A Randomized, Open-Label, Multicenter Controlled Study. J Clin Oncol 2019;37:2141-51.

30. Sun J, Yang L, Shi J, et al. Postoperative adjuvant IMRT for patients with HCC and portal vein tumor thrombus:

doi: $10.21037 /$ tro-20-15

Cite this article as: Huang LW, Lin HY, Chiou WY, Lee MS, Chen LC, Hung SK, Chang NY. Clinical-dosimetric parameters predicting non-classic radiation-induced liver disease in hepatocellular carcinoma patients with Barcelona Clinic Liver Cancer (BCLC) stage A-B: a retrospective study. Ther Radiol Oncol 2020;4:13.
An open-label randomized controlled trial. Radiother Oncol 2019;140:20-25.

31. Khozouz RF, Huq SZ, Perry MC. Radiation-induced liver disease. J Clin Oncol 2008;26:4844-5.

32. Guha C, Kavanagh BD. Hepatic radiation toxicity: avoidance and amelioration. Semin Radiat Oncol 2011;21:256-63.

33. Bae SH, Park HC, Yoon WS, et al. Treatment Outcome after Fractionated Conformal Radiotherapy for Hepatocellular Carcinoma in Patients with Child-Pugh Classification B in Korea (KROG 16-05). Cancer Res Treat 2019;51:1589-99. 